\title{
A loss of function variant in CASP7 protects against Alzheimer's disease in homozygous APOE $\varepsilon 4$ allele carriers
}

\author{
Kristin L. Ayers ${ }^{1}$, Uyenlinh L. Mirshahi ${ }^{2}$, Amr H. Wardeh², Michael F. Murray ${ }^{2}$, Ke Hao ${ }^{1}$, Benjamin S. Glicksberg ${ }^{1}$, \\ Shuyu Li', David J. Carey ${ }^{2}$ and Rong Chen ${ }^{1 *}$
}

From Varl-SIG at ISMB 2015

Dublin, Ireland 07 November 2015

\begin{abstract}
Background: Alzheimer's disease (AD) represents the most common form of dementia in elder populations with approximately 30 million cases worldwide. Genome wide genotyping and sequencing studies have identified many genetic variants associated with late-onset Alzheimer's disease (LOAD). While most of these variants are associated with increased risk of developing LOAD, only limited number of reports focused on variants that are protective against the disease.
\end{abstract}

Methods: Here we applied a novel approach to uncover protective alleles against AD by analyzing genetic and phenotypic data in Mount Sinai Biobank and Electronic Medical Record (EMR) databases.

Results: We discovered a likely loss-of-function small deletion variant in the caspase 7 (CASP7) gene associated with significantly reduced incidence of LOAD in carriers of the high-risk APOE $\varepsilon 4$ allele. Further investigation of four independent cohorts of European ancestry revealed the protective effect of the CASP7 variant against AD is most significant in homozygous APOE $\varepsilon 4$ allele carriers. Meta analysis of multiple datasets shows overall odds ratio $=0.45$ $(p=0.004)$. Analysis of RNA sequencing derived gene expression data indicated the variant correlates with reduced caspase 7 expression in multiple brain tissues we examined.

Conclusions: Taken together, these results are consistent with the notion that caspase 7 plays a key role in microglial activation driving neuro-degeneration during AD pathogenesis, and may explain the underlying genetic mechanisms that anti-inflammatory interventions in AD show greater benefit in APOE $\varepsilon 4$ carriers than non-carriers. Our findings inform potential novel therapeutic opportunities for $A D$ and warrant further investigations.

Keywords: Alzheimer's disease, Genetic variants, Protective alleles, CASP7, Resilience, Loss of function

\section{Background}

The prevalence of dementia in the Western population over age of 60 has been estimated to exceed $5 \%$. Alzheimer's disease $(\mathrm{AD})$ is the leading cause of dementia accounting for approximately 30 million cases worldwide $[1,2]$. The incidence and prevalence of $\mathrm{AD}$ is age dependent, doubling

\footnotetext{
* Correspondence: rong.chen@mssm.edu

${ }^{1}$ Department of Genetics and Genomic Sciences, Icahn Institute for Genomics and Multiscale Biology, Icahn School of Medicine at Mount Sinai, New York, NY 10029, USA

Full list of author information is available at the end of the article
}

every 5 years after age 65 [3]. As the proportion of population over 60 years of age continues to rise, the number of individuals developing $\mathrm{AD}$ will also increase, presenting a major health issue worldwide. Pathologically, AD is defined by extensive neuronal loss and accumulation of extracellular amyloid plaques formed by aggregation of $\beta$-amyloid (A $\beta)$ peptides and intracellular neurofibrillary tangles composed of hyper-phosphorylated tau proteins [4].

$\mathrm{AD}$ has a strong genetic component. It has been generally recognized that there are two major categories of $\mathrm{AD}$, familial early-onset $\mathrm{AD}$ (EOAD) and sporadic late-onset 
$\mathrm{AD}$ (LOAD). EOAD represents less than $1 \%$ of all $\mathrm{AD}$ cases and they are characterized by early-onset, often before age 60, and Mendelian inheritance occurring mostly in an autosomal dominant manner. Linkage analysis of EOAD families in the 80's and early 90's led to the discoveries of fully penetrant disease causing mutations in $A P P$ encoding $\beta$-amyloid precursor protein, PSEN1 and PSEN2 encoding components of the $\gamma$-secretase complex, presenilin 1 and presenilin 2 respectively [5]. In amyloidogenic pathway, APP, a transmembrane protein is first cleaved by a $\beta$-secretase, encoded by $B A C E 1$ and subsequently by the $\gamma$-secretase complex to form $A \beta$ peptides [6, 7]. Identification of disease causing mutations in APP, PSEN1, PSEN2 underscores the pathogenic role of the amyloidogenic pathway in AD development $[8,9]$.

Linkage studies, genome-wide association studies (GWAS), and recent whole exome sequencing (WES) have identified dozens of risk genes in $\operatorname{LOAD}[4,5,10]$. These risk genes have created a broader picture of pathways involved in AD pathogenesis. Several pathways have been highlighted by these genes, including cholesterol metabolism (APOE, CLU, ABCA7), immune response (CR1, CD33, MS4A, TREM2) and endocytosis (BIN1, PICALM, CD2AP, EPHA1, SORL1) [4]. Among $\mathrm{AD}$ risk genes and variants, $\mathrm{APOE}$ is the strongest risk factor. APOE has three isoforms determined by cysteineto-arginine substitutions at amino acid position 112 and 158, corresponding to two SNPs (rs429358 and rs7412 respectively) $[11,12]$. The three isoforms are referred as APOE2 (cys112, cys158), APOE3 (cys112, arg158) and APOE4 ( $\arg 158, \arg 158)$ with the corresponding alleles designated $\varepsilon 2, \varepsilon 3$, and $\varepsilon 4$ respectively [13]. APOE $\varepsilon 3$ is the most common isoform with 60-70 \% [14] allele frequency. APOE $\varepsilon 4$ allele is associated with increased AD risk in both familial EOAD and sporadic LOAD, with $2-5$ fold increased risk for heterozygous carriers and 12-15 fold increased risk for homozygous carriers in Caucasian populations $[5,15]$. These risks have been estimated for as a 5.7 fold increase in homozygous and no increased risk in heterozygotes in the African American population [16]. In Hispanics, this risk is estimated to be 2.2 fold in homozygotes with no increased risk in heterozygotes [16]. However, there is a greater prevalence of Alzheimer's and other dementias in African-Americans and Hispanics, suggesting other environmental or genetic factors are at play [17].

Elucidating the functional effects of naturally occurring genetic variants is one of the major challenges in genetic studies of human diseases [18]. With most of the genetic studies focused on variants associated with increased AD risks, there are a limited number of reports discussing variants that render protective effects against AD. The most notable example is the APP A673T mutation protecting against $\mathrm{AD}$ as well as cognitive decline in the elderly without $\mathrm{AD}$ due to $40 \%$ reduction in the formation of $A \beta$ peptides [19]. Missense variants in several other genes associated with lowered risk of AD or neuronal atrophy, including TREML2 [20], HMGCR [21], and REST [22], have recently been described. In this study, we applied a novel approach to discover $\mathrm{AD}$ protective variants by identifying genetic modifiers for $\mathrm{AD}$ risk in APOE high-risk $\varepsilon 4$ allele carriers. Genotyping data of approximately one million markers plus 37 million imputed SNPs in Mount Sinai Biobank [23] were analyzed and a small deletion variant (rs10553596) in the coding region of caspase 7 gene (CASP7) was found to be significantly associated with reduced incidence of $\mathrm{AD}$ and dementia in APOE $\varepsilon 4$ carriers. The protective effect of rs10553596 is observed in four independent LOAD cohorts. Interestingly, the protective effect of this CASP7 variant appears to be most significant in homozygous APOE $\varepsilon 4$ carriers. At gene expression level, eQTL analysis indicated that the rs10553596 variant is correlated with lowered caspase 7 expression. These results provide new insights into the underlying genetic mechanism of $\mathrm{AD}$ as well as opportunities for novel therapeutic strategies.

\section{Methods}

\section{Study participants or study cohorts}

We analyzed 6 datasets (Table 1): Mount Sinai Biobank ( 14 K individuals with genotype data), Geisinger Health System (GHS) MyCode Cohort (9856 unrelated individuals, [24]), GBAD (1588 individuals, http://www.ncbi.nlm. nih.gov/projects/gap/cgi-bin/study.cgi?study_id=phs00021 9.v1.p1), ADNI (2826 individuals, http://adni.loni.usc.edu), ADSP (10,939 individuals, https://www.niagads.org/adsp/ content/home), and ADGC (6065 individuals, http:// www.ncbi.nlm.nih.gov/projects/gap/cgi-bin/study.cgi?stud y_id=phs000372.v1.p1). ADSP is a large AD cohort including whole genome sequencing data. However, after downloading all APOE $\varepsilon 4$ homozygotes and filtering for a read count $\geq 10$ for the CASP7 variant, only one control remained (Table 1), and thus the data was not included in the meta-analysis.

\section{The Mount Sinai Biobank cohort}

Mount Sinai Biobank was established in 2007 in New York city and is distinguished among most biobanks because the participants are recruited from highly diverse communities, with the majority being either Hispanic or African American [23]. Currently it has collected DNA samples from more than 30,000 enrolled participants, and genotyping data has been generated for more than 14,000 patient samples using Illumina OmniExpressExome-8 v1.1 BeadChip that covers approximately one million genetic markers. Available clinical information for all the participants such as disease diagnosis, laboratory test results and medication history were obtained from Mount Sinai electronic medical record (EMR) databases. 
Table 1 The analyzed datasets in this study

\begin{tabular}{|c|c|c|c|c|c|c|}
\hline \multirow[t]{2}{*}{ Dataset } & \multirow[t]{2}{*}{ Samples } & \multirow[t]{2}{*}{ Cases } & \multirow[t]{2}{*}{ Controls } & \multicolumn{3}{|c|}{ APOE ع4 Homozygotes.... } \\
\hline & & & & All & Cases & Controls \\
\hline Mt. Sinai All & 13,710 & 238 & 5325 & 93 & 8 & 85 \\
\hline White & & & & 25 & 1 & 24 \\
\hline African American & & & & 37 & 3 & 34 \\
\hline Hispanic/Latino & & & & 28 & 4 & 24 \\
\hline GHS & 7645 & 287 & 7358 & 132 & 6 & 126 \\
\hline GBAD & 1588 & 806 & 782 & 103 & 92 & 11 \\
\hline ADNI & 2826 & 370 & 569 & 91 & 55 & 28 \\
\hline ADGC & 6065 & 3666 & 1604 & 456 & 419 & 25 \\
\hline ADSP & 10,939 & 6311 & 4628 & 145 & 96 & 1 \\
\hline TOTAL & 42,773 & 12,248 & 20,067 & 1020 & $572($ EUR > 60) & $190(E \cup R>60)$ \\
\hline
\end{tabular}

Some of these numbers may not sum due to various filtering steps. The final case/control totals for the APOE $\varepsilon 4$ homozygotes include only those analyzed in the meta-analysis, and thus do not include the Mt. Sinai or ADSP datasets

\section{Genotyping and imputation}

SNP genotypes were obtained using Illumina OmniEx pressExome-8 v1.1 BeadChip (Mt. Sinai), Illumina Infinium ExomeCore 12v1.0_B (GHS), Affymetrix $500 \mathrm{~K}$ array set (GBAD), Illumina Human610-Quad BeadChip and Illumina HumanOmniExpress BeadChip (https:/www.synapse.org/\#!Synapse:syn2290704/wiki/64710) (ADNI), and Illu mina Human660W-Quad/HumanOmniExpress (ADGC).

Quality control was performed on all genotype datasets prior to imputation. In the Mount Sinai Biobank, individuals with discordant sex, call rates below $98 \%$, or out-lying heterozygosity were removed. SNPs with call rates below $95 \%$ or with deviation from HardyWeinberg equilibrium (HWE) with $p$-value $<5 \mathrm{e}-5$ were also excluded. For the GHS dataset, genotypes were precleaned and imputed separately as previously described [25]. Samples with sex mismatches and low call rates and SNPs with low call rates were removed. For the GBAD Affymetrix $500 \mathrm{~K}$ dataset, the data was pre-QCed according to the protocol given with the data [26]. The GBAD data samples with call rates $<94 \%$ or gender mismatches were removed along with SNPs with HWE $p$ value $<5 \mathrm{e}-7$ or call rate $<95 \%$ (99\% for MAF $<0.05)$. For the ADNI dataset, samples with call rates $<98 \%$, population outliers, and relateds were removed along with SNPs with HWE $p$-value $<0.001$, minor allele frequency $(\mathrm{MAF})<0.05$, or call rate $<98 \%$ according the protocol at http://adni.loni.usc.edu/ and https://www.synapse.org/\#!Synapse:syn2290704/wiki/64710. For the ADGC dataset, data samples with call rates $<99 \%$, gender mismatches, and individuals with outlying heterozygosity were removed along with SNPs with HWE $p$-value $<1 \mathrm{e}-10$, call rate $<98 \%$, and $\mathrm{MAF}<1 \%$.

All genotype datasets were prephased using SHAPEITv2 [27] and imputation was performed with IMPUTE2 [28] using the 1000 genomes integrated variant set as the reference panel. The Mount Sinai Biobank, the GHS dataset, the ADGC dataset, and the GBAD dataset were imputed with the phase III haplotype reference panel, while the ADNI dataset was pre-imputed with the phase I panel. The imputation info score for the CASP7 variant rs10553596 was 0.97 in the Mount Sinai Biobank, and 0.99 in the GBAD, ADGC and GHS cohorts. For the GHS, GBAD, ADGC, and ADNI datasets, individuals who had a posterior probability of less than 0.9 for the most likely genotype were excluded from the analysis. The CASP7 variant rs10553596 was imputed in all datasets.

\section{APOE $\varepsilon 4$ status}

Individuals who have the $\mathrm{C} / \mathrm{C}$ genotype at rs429358 are obligate homozygous $\varepsilon 4$, and those who harbor the T/T genotype as rs7412 are obligate non- $\varepsilon 4$-carriers. Individuals who were $\mathrm{C} / \mathrm{C}$ at $\mathrm{rs} 429358$ and $\mathrm{T} / \mathrm{T}$ at rs7412 were removed from the analysis, as this haplotype is not observed (and it is thus due to genotype error or incorrect genotype imputation). In the Mt. Sinai Biobank, rs7412 was genotyped while rs429358 was imputed with imputation info of 0.96. Two individuals were excluded from the analysis due to an inconsistent haplotype. In the GHS cohort, 2033 individuals were identified as APOE $\varepsilon 4$ carriers (rs7412 was genotyped and rs429358 was imputed), of which 812 carry at least 1 allele for CASP7 rs10553596. In the GBAD dataset, rs429358 and rs7412 were genotyped independently. APOE $\varepsilon 4$ status and the CASP7 rs10553596 genotype could be estimated for 1423 of the 1588 individuals. The ADNI dataset included the number of copies of the APOE $\varepsilon 4$ allele (both rs7412 and rs429358 were genotyped). There were 919 individuals of European descent with APOE $\varepsilon 4$ status. The ADGC dataset provided the two APOE alleles for each individual. 


\section{Phenotype determination}

Since EOAD occurs in individuals before the age of 60 , we selected individuals older than 60 in all cohorts as one of the criteria to define LOAD. For both the Mount Sinai Biobank and GHS EMR data, AD cases were selected using ICD9 billing codes 290, 294, and 331 and all their subtypes (excluding Reye's syndrome (331.81) and alcohol induced dementia (291.2)) from electronic medical records (EMR). Cases were defined as individuals with first record of $\mathrm{AD} /$ dementia diagnoses above age 60 . Controls were those over 60 year of age at their last recorded visit with no records including ICD9 290, 294, and 331. In the GBAD dataset, LOAD cases and controls were defined according to the protocol at http:// www.ncbi.nlm.nih.gov/projects/gap/cgi-bin/study.cgi?study_id=phs000219.v1.p1. For the ADNI dataset, visits were recorded for patients in 3 categories: NL (normal), MCI (mild cognitive impairment), or Dementia. For these individuals, we used the most severe diagnosis recorded. Thus, individuals recorded as NL and MCI were considered controls, and those recorded as Dementia were considered cases. Only individuals recorded as 'White' and 'Not Hispanic/Latino' and who were over the age of 60 were analyzed. The ADGC dataset includes individuals with normal phenotype, AD, or MCI. Individuals classed as normal/MCI who were over 60 years of age at the last visit were considered controls, and $\mathrm{AD}$ patients with initial diagnosis after age 60 were considered cases. Only individuals recorded as "White" and "Not Hispanic/Latino" who clustered together in PC analysis were analyzed.

\section{Meta analysis}

A weighted fixed-effect meta-analysis was performed in the $\mathrm{R}$ package metafor assuming a dominant model for the CASP7 rs10553596 variant in homozygous APOE \&4 carriers, heterozygous $\varepsilon 4$ carriers, and non-carriers (adding a half count to any zero count cells). To test for any possible population stratification issues within the European cohorts, we included the first 3 principle components (PCs) as covariates in a logistic regression model for the APOE $\varepsilon 4$ homozygotes for each of the ADNI, ADGC, and GBAD datasets. The PCs were not statistically significant nor did they have a notable impact on the estimated odds ratio.

\section{Results and discussion}

Identification of genetic variants associated with reduced AD prevalence in APOE $\varepsilon 4$ carriers

We first performed an exploratory analysis in the Mount Sinai Biobank to find protective alleles for APOE $\varepsilon 4$ homozygotes, who are at high risk for AD. The SNP rs429358, used to determine APOE $\varepsilon 4$ status, is well known for being difficult to genotype and failed QC on our array. The imputed genotypes were used to estimate the APOE $\varepsilon 4$ status. Though this variant has overall high imputation quality, genotype imputation is more difficult in populations of African ancestry, due to lower linkage disequilibrium (LD) and higher genetic diversity. If we were to use a cutoff of genotype posterior probability > 0.9 for the imputed rs429358 SNPs, this would result in only 29 individuals above age of 60 being assigned as homozygous APOE $\varepsilon 4$ carriers, of which only 2 were diagnosed with $\mathrm{AD} /$ dementia. We decided to use a loose threshold of genotype posterior probability $(>0.5)$ for the APOE SNP rs429358 in order to increase sample size for a more powerful test of significance. We applied this threshold to all tested imputed variants. Using ICD9 billing codes for AD and dementia diagnosis (290, 294 and 331 ), we had $8 \mathrm{AD} /$ dementia patients and 72 controls. With a one-sided Fisher's exact test, we found 12 annotated loss-of-function variants with $p$-value $<0.05$ that were protective for $\mathrm{AD} /$ dementia. The top two variants were in SLC22A24 (a putative ion transporter with unknown functional relevance to AD) and MUC19 (a gene known to give false positive associations due to its size). The third most significant variant was in CASP7, rs10553596, with an odds ratio of 0.080 and $p$-value of 0.0062. In this variant, two nucleotides (TT) is deleted in CASP7 coding region resulted in leucine to serine change at amino acid position 44 (mRNA accession: NM_001267057.1; protein accession: NP_001253986.1). In addition, the TT deletion causes a premature termination codon downstream at amino acid position 133. Therefore, it is highly likely this is a loss-of-function variant. The association of this variant to AD in the APOE $\varepsilon 4$ heterozygotes (odds ratio $=0.75, p$-value $=0.37$ ) suggests that the protective effect of rs10553596 against AD in all APOE $\varepsilon 4$ carriers might be present mainly in the APOE $\varepsilon 4$ homozygous population. Rs10553596 is a relatively common variant in general population, with differing allele frequencies amongst the different 1000 genomes populations (Additional file 1: Figure S1).

Next, we reinvestigated rs10553596 with a more stringent threshold for the imputed variants to determine if an association still holds. To increase the size of the test group, we performed a case-control analysis of genotypes between those diagnosed with $\mathrm{AD}$ or dementia and those without among APOE $\varepsilon 4$ carriers. Using the combined posterior probability of homozygous and heterozygous rs429358 and the information from the other genotyped APOE SNP rs7412, we can be fairly confident whether or not an individual carries at least one copy of APOE $\varepsilon 4$ (we can apply the same rational to the imputed variant CASP7 variant rs10553596 since we are analyzing a dominant model). With a cutoff for the combined posterior probability $>0.8,842$ individuals above age of 60 and in the top three largest ethnic groups were identified as APOE $\varepsilon 4$ carriers (Table 2a). As the control group, 3666 were identified as non-APOE $\varepsilon 4$ carriers 
Table 2 Association between CASP7 rs10553596 and AD/ dementia status in APOE $\varepsilon 4$ allele carriers (A), and non-APOE $\varepsilon 4$ allele carriers (B), stratified by ethnicity in Mount Sinai Biobank

\begin{tabular}{|c|c|c|c|c|c|}
\hline \multicolumn{6}{|c|}{ A. Carriers: Overall $P$-value $=0.031, \mathrm{OR}=0.56(95 \% \mathrm{Cl} 0.00-0.94)$} \\
\hline Ethnicity & Phenotype & Ref-Ref & Alt-* & $P$-value & Odds Ratio \\
\hline \multirow[t]{2}{*}{ European } & Controls & 137 & 141 & 0.25 & 0.49 \\
\hline & AD/dementia & 6 & 3 & & \\
\hline \multirow[t]{2}{*}{ African } & Controls & 68 & 145 & 0.19 & 0.54 \\
\hline & AD/dementia & 7 & 8 & & \\
\hline \multirow[t]{2}{*}{ Hispanic } & Controls & 155 & 146 & 0.12 & 0.56 \\
\hline & AD/dementia & 17 & 9 & & \\
\hline \multicolumn{6}{|c|}{ B. Non-Carriers: Overall $P$-value $=0.060, \mathrm{OR}=0.77(95 \% \mathrm{Cl} 0.00-1.01)$} \\
\hline Ethnicity & Phenotype & Ref-Ref & Alt-* & $P$-value & Odds Ratio \\
\hline \multirow[t]{2}{*}{ European } & Controls & 588 & 637 & 0.43 & 0.83 \\
\hline & AD/dementia & 10 & 9 & & \\
\hline \multirow[t]{2}{*}{ African } & Control & 233 & 453 & 0.24 & 0.74 \\
\hline & AD/dementia & 14 & 20 & & \\
\hline \multirow[t]{2}{*}{ Hispanic } & Control & 867 & 731 & 0.17 & 0.80 \\
\hline & AD/dementia & 62 & 42 & & \\
\hline
\end{tabular}

and are above age of 60 (Table 2b). 50 of the 842 APOE $\varepsilon 4$ carriers, and 157 of the 3666 individuals in the control group were diagnosed with $\mathrm{AD}$ or dementia. This represents an odds ratio of 1.4 fold for AD risk in APOE $\varepsilon 4$ carriers. The result shows that presence of at least one copy of the rs10553596 alternate allele significantly lowers the $\mathrm{AD}$ risk (odds ratio $=0.56, \mathrm{p}=0.031$ ) in APOE $\varepsilon 4$ carriers (Table 2a). However, when the same analysis was performed on non-APOE $\varepsilon 4$ carriers, no significant association between rs10553596 and reduced $\mathrm{AD}$ risk was detected (Table $2 \mathrm{~b}$ ) although a trend was observed. This suggests that rs10553596 is not protective for $\mathrm{AD}$ by its own, but is most likely a modifier for $\mathrm{APOE} \varepsilon 4$ alleles on $\mathrm{AD}$ risk.

\section{Evaluation of rs10553596 as an AD protective variant}

CASP7 encodes a member of the cysteine-aspartic acid protease (caspase) family. Sequential activation of caspases plays a central role in the execution-phase of cell apoptosis. However, the function of caspase 7 is beyond its role in apoptosis. For example, it has been illustrated that microglia cell activation by inflammatory signals are mediated through caspase cascade involving caspase 3 and caspase 7 , without triggering apoptosis [29]. Microglial are resident immune cells in brain acting as the main form of immune response in central nervous system (CNS). Abnormal microglial activation causes neurotoxicity and plays a predominant role in both $\mathrm{AD}$ and Parkinson's disease (PD) pathogenesis. A recent study has shown that a LD haplotype block containing the CASP7 gene is associated with reduced AD risk in Caribbean Hispanic populations [30].
We then attempted to replicate our findings in five independent cohorts (Table 1). (1) The GBAD study [26, 31], with French Canadian ancestry, (2) the ADNI study [32], (3) the GHS MyCode study [24], (4) the ADGC study [33], and (5) the ADSP study (http://www.ncbi.nlm.nih.gov/projects/gap/cgi-bin/study.cgi?study_id=phs000572. v1.p1). We assigned each individual into one of the following three populations: APOE $\varepsilon 4$ homozygotes, APOE $\varepsilon 4$ heterozygotes and non-APOE $\varepsilon 4$ carriers based on their APOE genotypes. For the ADSP WGS dataset, after genotype calling, we were left with only one APOE $\varepsilon 4$ homozygote control, and thus the data was not included in the meta-analysis. Because these cohorts are of primarily of European ancestry, one can achieve higher accuracy of genotype imputation due to longer stretches of LD and lower genetic diversity. The protective effect of CASP7 variant rs10553596 was then assessed for individuals with genotype posterior probability of $>0.9$ for any genotype in each of the three sub-populations (APOE $\varepsilon 4$ homozygotes, APOE $\varepsilon 4$ heterozygotes and non-APOE $\varepsilon 4$ carriers). The combined meta-analysis $p$-value (odds ratio) for these four cohorts was 0.004 (0.45) in homozygous APOE $\varepsilon 4$ carriers (Fig. 1a), indicating a significant protective effect of CASP7 rs1055359. In contrast, this protective effect of the rs10553596 variant is not observed in APOE $\varepsilon 4$ heterozygotes (Fig. 1b) and non-carriers (Fig. 1c).

Interestingly, when we interrogated a centralized genetic variant repository (DIVAS) we built that included genotyping and sequencing data of 150,000 individuals from multiple disease cohorts [34], we observed that allele frequency of rs10553596 is significantly lower in familial Amyotrophic lateral sclerosis (ALS) at $5 \%$ in comparison to $19-50 \%$ in healthy populations (Additional file 1: Figure S2), implicating a protective effect of this variant against ALS, another neuro-degenerative disease. The familiar ALS cohort was downloaded from dbGap with accession phs000101.v4.p1 $[35,36]$. Although this observation requires further validation given rs10553596 is an indel variant and the technical challenges associated with indel calling, it is consistent with our findings on the protective effect of rs10553596 in LOAD. We further assessed the effect of the rs10553596 variant on CASP7 gene expression through eQTL analysis. As demonstrated in Table 3, the alternate allele of rs10553596 is significantly associated with lowered CASP7 expression in the tissues we examined, including several brain tissues such as prefrontal cortex, visual cortex and cerebellum. This supports that rs10553596 is a loss of function variant for CASP7.

African American and Hispanic APOE $\varepsilon 4$ carriers have a lower risk of developing Alzheimer's or dementia than European carriers. The allele frequencies of the CASP7 variant rs10553596 differ among the 1000 genomes ethnicities: $34-48 \%$ in African populations, $21-32 \%$ in South 


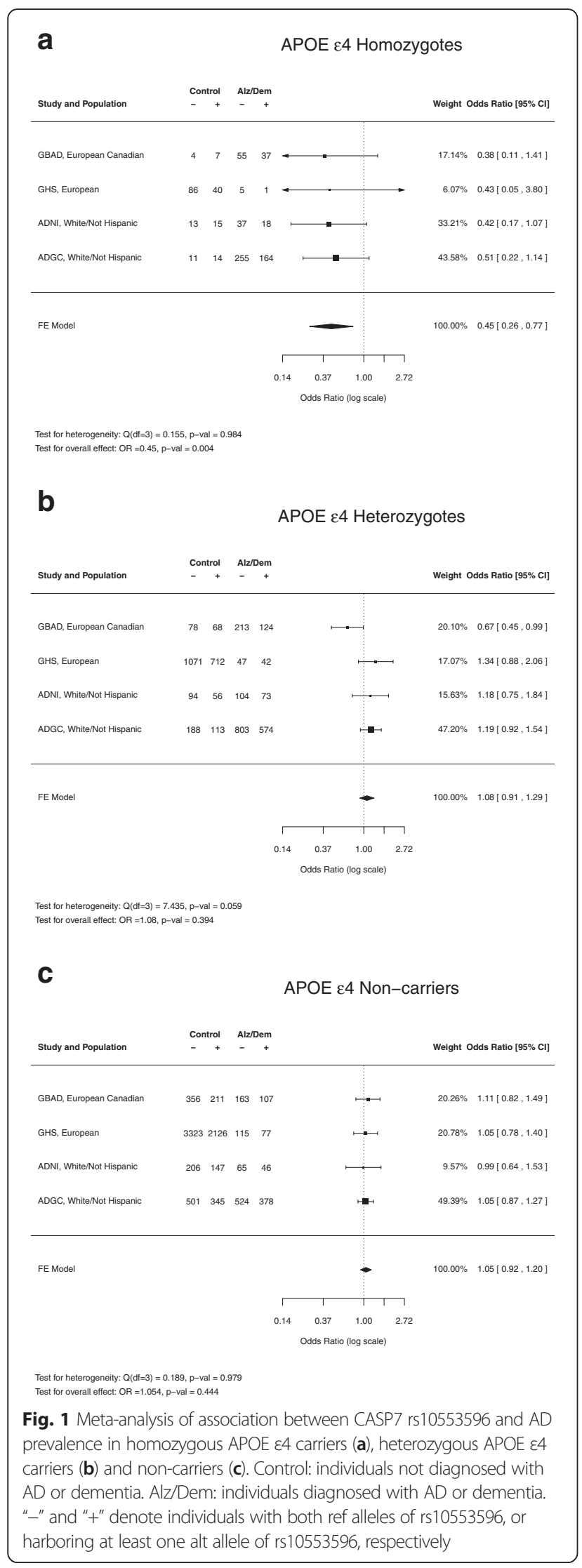

Asian populations, 19-29 \% in European populations, 2128 \% in East Asian populations, and 15-28 \% in American populations (Additional file 1: Figure S1). The higher allele frequency of rs10553596 in African populations might explain why APOE $\varepsilon 4$ African Americans have a lower risk for Alzheimer's than Europeans [17]. It is identified in this study, possibly due to the unique composition of population in Mount Sinai Biobank with the majority being either African American or Hispanic [23], although we were able to confirm the protective effect of rs10553596 against AD in four European cohorts. If we plot the frequency of rs10553596 versus the estimated APOE $\varepsilon 4$ frequencies, we see a general positive correlation $\left(r^{2}=0.42\right)$, which may reflect selective pressure (Additional file 1: Figure S1). We also realize that diversity of the frequency of this marker and the varying risks amongst ethnicities may inflate association due to subtle population stratification issues. While this is definitely a possibility in the Mount Sinai Biobank due to complex admixtures, it is far less likely to be a major factor in the more homogenous cohorts of European descent (principle component analysis supports this conclusion, see Methods).

The protective effect of rs10553596 against LOAD is only observed in APOE $\varepsilon 4$ homozygote populations (Fig. 1). It has been well documented that APOE binds to $A \beta$ and influences the clearance of soluble $A \beta$ and the $A \beta$ aggregation [4]. However it was unclear whether APOE genotype affects A $\beta$-plaque-associated neuro-inflammation until a recent study demonstrated that in addition to altering morphological profiles of $A \beta$ deposition, APOE genotype influences $\mathrm{A} \beta$-induced glial activation in cortex [37], a key step leading to neuro-inflammation in AD pathogenesis. Given the critical role of caspase 7 in microglial activation and recent findings implicating APOE $\varepsilon 4$ alleles in modulating neuroinflammatory responses in $\mathrm{AD}$ development, it is only fitting that the loss-of-function alt allele of rs10553596 in CASP7 exhibited protective effect against AD in APOE high-risk $\varepsilon 4$ carrier populations. Moreover, the protective effect of a CASP7 loss-of-function variant against AD only in APOE $\varepsilon 4$ homozygotes may explain the clinical observation that anti-inflammatory interventions in $\mathrm{AD}$ show greater benefit in APOE $\varepsilon 4$ carriers as opposed to non-carriers [38]. As pan-caspase inhibitors have been under development for several autoimmune diseases [39], our discovery has potentially significant clinical relevance implicating novel therapeutic opportunities for AD.

We also recognize the limitations in our study. Limited sample size is an inherent challenge. Two of the 6 datasets, Mount Sinai Biobank and Geisinger Health System (GHS) MyCode Cohort, are population-based data that is not focused on AD. Therefore, the frequency of APOE $\varepsilon 4$ homozygous carriers is equal to the population frequency of approximately 1-2 \%. Multiple filtering 
Table 3 rs10553596-c allele is associated with lower CASP7 gene expression level

\begin{tabular}{lllclc}
\hline Tissue & Reporter ID & Effective allele & eQTL P-value & eQTL direction & Sample size \\
\hline Lung & $100139172 \_T G I$ at & c & $2.6 \mathrm{e}-44$ & $\downarrow$ & 1111 \\
Prefrontal cortex & 10023817180 & c & $1.5 \mathrm{e}-21$ & $\downarrow$ & 583 \\
Visual cortex & 10023817180 & c & $4.0 \mathrm{e}-11$ & $\downarrow$ & 409 \\
Cerebellum & 10023817180 & c & $5.7 \mathrm{e}-12$ & $\downarrow$ & 496 \\
Liver & 10023817180 & c & $2.1 \mathrm{e}-23$ & $\downarrow$ & 563 \\
Omental fat & 10023817180 & c & $2.6 \mathrm{e}-62$ & $\downarrow$ & 675 \\
Subcutaneous fat & 10023817180 & c & $1.6 \mathrm{e}-29$ & $\downarrow$ & 611 \\
\hline
\end{tabular}

Expressional QTLs (eQTLs) between rs10553596 and CASP7 expression level were reported in large tissue collections, including lung [40], brain regions [41], liver [42] and fat tissues [42]. In lung eQTL study, Affymetrix HU133 array was used; and in other eQTL studies, Agilent Human44K1.1 array were employed

steps throughout the analyses (see Methods) further reduced the sample size. In addition, there are a significantly smaller number of $\mathrm{AD}$ patients than controls. In contrast, the $4 \mathrm{AD}$ datasets (GBAD, ADNI, ADGC and ADSP) have significantly larger number of $\mathrm{AD}$ patients than controls among APOE \&4 homozygous carriers since the APOE $\varepsilon 4$ allele is enriched in $\mathrm{AD}$ populations. Unbalanced $\mathrm{AD}$ vs. control sample size poses a great challenge to achieve the level of statistical significance reported in typical GWAS studies with balanced case vs. control populations of thousands to tens of thousands in sample size. Even with an original sample size of nearly 40,000 individuals, we were left with $<1000$ homozygous APOE $\varepsilon 4$ individuals for the metaanalysis (Table 1; Fig. 1). Another limitation is that we define age 60 , a commonly used threshold as a cutoff for LOAD. It is likely that some of the individuals in the control population will develop $\mathrm{AD}$ as they age. Therefore, our analyses suggest that the CASP7 variant rs10553596 protects against AD or simply delays the age of onset. As more AD genotyping and genomic sequencing data from large consortium become available, we can define a more stringent age threshold or use age of onset as a quantitative trait in an $\mathrm{AD}$ population to distinguish these possibilities.

\section{Conclusions}

In this study, we analyzed individuals who are APOE $\varepsilon 4$ risk allele carriers but are resilient to Alzheimer's disease, and discovered a novel AD protective genetic variants modifying AD risk in APOE \&4 risk allele carriers. Our analysis of Mount Sinai Biobank cohort led to the identification of a likely loss-of-function variant rs10553596 in CASP7 associated with significantly reduced AD incidence in elder populations. The protective effect of rs10553596 against AD was observed in four independent cohorts of European descent with similar odds ratios, and the overall effect in these cohorts was statistically significant. In addition, we show rs10553596 is associated in cis with lowered caspase 7 gene expression. Taken together, these results are consistent with the notion that caspase 7 plays a critical role in mediating microglial activation in CNS that triggers neurotoxicity and AD pathogenesis.

\section{Ethics approval and consent to participate}

The study was approved by the Mount Sinai Institutional Review Board (IRB), and conducted in accordance with the Declaration of Helsinki and Good Clinical Practice (GCP). Informed consent was obtained from all participants in Mount Sinai Biobank cohort and Geisinger Health System (GHS) MyCode Cohort.

\section{Consent for publication}

Not applicable.

\section{Availability of data and material}

Not applicable.

\section{Additional file}

Additional file 1: Figure S1. Relationship between the frequency of rs10553596 in CASP7 and APOE e4 allele frequency. (A) Variant frequencies across the 1000 genome phase 3 ethnic groups for rs 10553596 and the APOE $\varepsilon 4$ allele, The ethnic groups are color coded by continent/region. (B) The frequency of rs10553596 versus the APOE $\varepsilon 4$ allele. Figure S2. Frequency bar chart showing the variant frequencies across all DIVAS disease and control cohorts for rs10553596. The y axis shows the allele frequencies. Blue bars represent healthy cohorts with different ethnicities. Red bars present diseased cohorts. FALS stands for familial Amyotrophic lateral sclerosis. (PDF $134 \mathrm{~kb}$ )

\section{Competing interests}

The authors declare that they have no competing interests.

\section{Authors' contributions}

$R C$ designed the study. SL and RC supervised the study. KLA, ULM, AHW, MFM, KH, BSG performed data analysis. KLA, ULM, MFM, SL, DJC, RC interpreted the results. KLA and SL wrote the manuscript. ULM and RC revised the manuscript. All authors read and approved the final manuscript.

\section{Acknowledgement}

This work was supported in part through the computational resources and staff expertise provided by the Department of Scientific Computing at the Icahn School of Medicine at Mount Sinai. We acknowledge the suggestions from Brian Naughton at 23 \& me, and conversations with Alison Goate, Giulio Pasinetti, Lap Ho, and Eric Schadt from Icahn School of Medicine. We thank Wei-Yi Cheng for his work on the preliminary GWAS analysis. 


\section{Declarations}

This study and the publication were funded by Icahn School of Medicine at Mount Sinai. This article has been published as part of BMC Genomics Volume 17 Supplement 2, 2016: Proceedings of Varl-SIG 2015: Identification and annotation of genetic variants in the context of structure, function, and disease. The full contents of the supplement are available online at https://bmcgenomics.biomedcentral.com/articles/supplements/volume-17-supplement-2.

\section{Funding}

This study and the publication were funded by Icahn School of Medicine at Mount Sinai.

\section{Author details}

'Department of Genetics and Genomic Sciences, Icahn Institute for Genomics and Multiscale Biology, Icahn School of Medicine at Mount Sinai, New York, NY 10029, USA. 'Geisinger Clinic, Danville, PA 17822, USA.

Published: 23 June 2016

\section{References}

1. Reitz C, Brayne C, Mayeux R. Epidemiology of Alzheimer disease. Nat Rev Neurol. 2011;7(3):137-52.

2. Reitz C, Mayeux R. Alzheimer disease: epidemiology, diagnostic criteria, risk factors and biomarkers. Biochem Pharmacol. 2014;88(4):640-51.

3. Qiu C, Kivipelto M, von Strauss E. Epidemiology of Alzheimer's disease: occurrence, determinants, and strategies toward intervention. Dialogues Clin Neurosci. 2009;11(2):111-28.

4. Karch CM, Goate AM. Alzheimer's disease risk genes and mechanisms of disease pathogenesis. Biol Psychiatry. 2015;77(1):43-51.

5. Chouraki V, Seshadri S. Genetics of Alzheimer's disease. Adv Genet. 2014;87:245-94.

6. Haass C, Kaether C, Thinakaran G, Sisodia S. Trafficking and proteolytic processing of APP. Cold Spring Harb Perspect Med. 2012;2(5):a006270.

7. Zhang H, Ma Q, Zhang YW, Xu H. Proteolytic processing of Alzheimer's beta-amyloid precursor protein. J Neurochem. 2012;120 Suppl 1:9-21.

8. Barage SH, Sonawane KD. Amyloid cascade hypothesis: pathogenesis and therapeutic strategies in Alzheimer's disease. Neuropeptides. 2015;52:1.

9. De-Paula VJ, Radanovic M, Diniz BS, Forlenza OV. Alzheimer's disease. Sub-cellular biochemistry. 2012;65:329-52.

10. Karch CM, Cruchaga C, Goate AM. Alzheimer's disease genetics: from the bench to the clinic. Neuron. 2014;83(1):11-26.

11. Zannis VI, Breslow JL. Human very low density lipoprotein apolipoprotein E isoprotein polymorphism is explained by genetic variation and posttranslational modification. Biochemistry. 1981;20(4):1033-41.

12. Zannis VI, Just PW, Breslow JL. Human apolipoprotein E isoprotein subclasses are genetically determined. Am J Hum Genet. 1981;33(1):11-24.

13. Zannis VI, Breslow JL, Utermann G, Mahley RW, Weisgraber KH, Havel RJ, Goldstein JL, Brown MS, Schonfeld G, Hazzard WR, et al. Proposed nomenclature of apoE isoproteins, apoE genotypes, and phenotypes. J Lipid Res. 1982;23(6):911-4.

14. Yu JT, Tan L, Hardy J. Apolipoprotein E in Alzheimer's disease: an update. Annu Rev Neurosci. 2014;37:79-100.

15. Genin E, Hannequin D, Wallon D, Sleegers K, Hiltunen M, Combarros O, Bullido MJ, Engelborghs S, De Deyn P, Berr C, et al. APOE and Alzheimer disease: a major gene with semi-dominant inheritance. Mol Psychiatry. 2011;16(9):903-7.

16. Farrer LA, Cupples LA, Haines JL, Hyman B, Kukull WA, Mayeux R, Myers RH, Pericak-Vance MA, Risch N, van Duijn CM. Effects of age, sex, and ethnicity on the association between apolipoprotein E genotype and Alzheimer disease. A meta-analysis. APOE and Alzheimer Disease Meta Analysis Consortium. JAMA. 1997;278(16):1349-56.

17. Gurland BJ, Wilder DE, Lantigua R, Stern Y, Chen J, Killeffer EH, Mayeux R. Rates of dementia in three ethnoracial groups. Int I Geriatr Psychiatry. 1999; 14(6):481-93.

18. Hecht M, Bromberg Y, Rost B. Better prediction of functional effects for sequence variants. BMC Genomics. 2015;16 Suppl 8:S1.

19. Jonsson T, Atwal JK, Steinberg S, Snaedal J, Jonsson PV, Bjornsson S, Stefansson H, Sulem P, Gudbjartsson D, Maloney J, et al. A mutation in APP protects against Alzheimer's disease and age-related cognitive decline. Nature. 2012:488(7409):96-9.
20. Benitez BA, Jin SC, Guerreiro R, Graham R, Lord J, Harold D, Sims R, Lambert JC, Gibbs JR, Bras J, et al. Missense variant in TREML2 protects against Alzheimer's disease. Neurobiol Aging. 2014;35(6):1510. e1519-1526.

21. Leduc V, De Beaumont L, Theroux L, Dea D, Aisen P, Petersen RC, Dufour R, Poirier J. HMGCR is a genetic modifier for risk, age of onset and $\mathrm{MCl}$ conversion to Alzheimer's disease in a three cohorts study. Mol Psychiatry. 2015;20(7):867-73.

22. Nho K, Kim S, Risacher SL, Shen L, Corneveaux JJ, Swaminathan S, Lin H, Ramanan VK, Liu Y, Foroud TM, et al. Protective variant for hippocampal atrophy identified by whole exome sequencing. Ann Neurol. 2015;77(3):547-52.

23. Streicher SA, Sanderson SC, Jabs EW, Diefenbach M, Smirnoff M, Peter I, Horowitz CR, Brenner B, Richardson LD. Reasons for participating and genetic information needs among racially and ethnically diverse biobank participants: a focus group study. J Community Genet. 2011;2(3):153-63.

24. Carey D, Fetterolf S, Davis F, Faucett W, Kircher H, Mirshahi U, Murray M, Smelser D, Gerhard G, Ledbetter D. The Geisinger MyCode Community Health Initiative: an electronic health record-linked biobank for Precision Medicine research. Genet Med. 2015. doi: 10.1038/gim.2015.187

25. Verma SS, de Andrade M, Tromp G, Kuivaniemi H, Pugh E, Namjou-Khales B, Mukherjee S, Jarvik GP, Kottyan LC, Burt A, et al. Imputation and quality control steps for combining multiple genome-wide datasets. Front Genet. 2014;5:370.

26. Li H, Wetten S, Li L, St Jean PL, Upmanyu R, Surh L, Hosford D, Barnes MR, Briley JD, Borrie M, et al. Candidate single-nucleotide polymorphisms from a genomewide association study of Alzheimer disease. Arch Neurol. 2008; 65(1):45-53.

27. Delaneau O, Zagury JF, Marchini J. Improved whole-chromosome phasing for disease and population genetic studies. Nat Methods. 2013;10(1):5-6.

28. Howie B, Marchini J, Stephens M. Genotype imputation with thousands of genomes. G3 (Bethesda, Md). 2011;1(6):457-70.

29. Burguillos MA, Deierborg T, Kavanagh E, Persson A, Hajji N, GarciaQuintanilla A, Cano J, Brundin P, Englund E, Venero JL, et al. Caspase signalling controls microglia activation and neurotoxicity. Nature. 2011; 472(7343):319-24.

30. Shang Z, Lv H, Zhang M, Duan L, Wang S, Li J, Liu G, Ruijie Z, Jiang Y. Genome-wide haplotype association study identify TNFRSF1A, CASP7, LRP1B, CDH1 and TG genes associated with Alzheimer's disease in Caribbean Hispanic individuals. Oncotarget. 2015;6(40):42504-14.

31. Filippini N, Rao A, Wetten S, Gibson RA, Borrie M, Guzman D, Kertesz A, LoyEnglish I, Williams J, Nichols T, et al. Anatomically-distinct genetic associations of APOE epsilon4 allele load with regional cortical atrophy in Alzheimer's disease. Neurolmage. 2009;44(3):724-8.

32. Weiner MW, Veitch DP, Aisen PS, Beckett LA, Cairns NJ, Cedarbaum J, Donohue MC, Green RC, Harvey D, Jack CR, Jr, et al. Impact of the Alzheimer's Disease Neuroimaging Initiative, 2004 to 2014. Alzheimer's \& dementia: the journal of the Alzheimer's Association. 2015;11(7):865-84.

33. Naj AC, Jun G, Beecham GW, Wang LS, Vardarajan BN, Buros J, Gallins PJ, Buxbaum JD, Jarvik GP, Crane PK, et al. Common variants at MS4A4/ MS4A6E, CD2AP, CD33 and EPHA1 are associated with late-onset Alzheimer's disease. Nat Genet. 2011;43(5):436-41.

34. Cheng WY, Hakenberg J, Li SD, Chen R. DIVAS: a centralized genetic variant repository representing 150000 individuals from multiple disease cohorts. Bioinformatics. 2015;32:151.

35. Johnson JO, Mandrioli J, Benatar M, Abramzon Y, Van Deerlin VM, Trojanowski JQ, Gibbs JR, Brunetti M, Gronka S, Wuu J, et al. Exome sequencing reveals VCP mutations as a cause of familial ALS. Neuron. 2010;68(5):857-64.

36. Johnson JO, Pioro EP, Boehringer A, Chia R, Feit H, Renton AE, Pliner HA, Abramzon Y, Marangi G, Winborn BJ, et al. Mutations in the Matrin 3 gene cause familial amyotrophic lateral sclerosis. Nat Neurosci. 2014;17(5):664-6.

37. Rodriguez GA, Tai LM, LaDu MJ, Rebeck GW. Human APOE4 increases microglia reactivity at Abeta plaques in a mouse model of Abeta deposition. J Neuroinflammation. 2014;11:111.

38. Szekely CA, Breitner JC, Fitzpatrick AL, Rea TD, Psaty BM, Kuller LH, Zandi PP. NSAID use and dementia risk in the Cardiovascular Health Study: role of APOE and NSAID type. Neurology. 2008;70(1):17-24.

39. Mackenzie SH, Schipper JL, Clark AC. The potential for caspases in drug discovery. Curr Opin Drug Discov Devel. 2010;13(5):568-76.

40. Hao K, Bosse Y, Nickle DC, Pare PD, Postma DS, Laviolette M, Sandford A, Hackett TL, Daley D, Hogg JC, et al. Lung eQTLs to help reveal the molecular underpinnings of asthma. PLoS Genet. 2012;8(11):e1003029. 
41. Zhang B, Gaiteri C, Bodea LG, Wang Z, McElwee J, Podtelezhnikov AA, Zhang C, Xie T, Tran L, Dobrin R, et al. Integrated systems approach identifies genetic nodes and networks in late-onset Alzheimer's disease. Cell. 2013:153(3):707-20.

42. Greenawalt DM, Dobrin R, Chudin E, Hatoum IJ, Suver C, Beaulaurier J, Zhang B, Castro V, Zhu J, Sieberts SK, et al. A survey of the genetics of stomach, liver, and adipose gene expression from a morbidly obese cohort. Genome Res. 2011;21(7):1008-16.

Submit your next manuscript to BioMed Central and we will help you at every step:

- We accept pre-submission inquiries

- Our selector tool helps you to find the most relevant journal

- We provide round the clock customer support

- Convenient online submission

- Thorough peer review

- Inclusion in PubMed and all major indexing services

- Maximum visibility for your research

Submit your manuscript at www.biomedcentral.com/submit 